\title{
Coherent charge transport through molecular wires: Influence of strong Coulomb repulsion
}

\author{
Franz J. Kaiser, Michael Strass, Sigmund Kohler, Peter Hänggi * \\ Institut für Physik, Universität Augsburg, Universitätsstraße 1, D-86135 Augsburg, Germany
}

Received 10 June 2005; accepted 12 August 2005

Available online 19 September 2005

\begin{abstract}
We derive a master equation for the electron transport through molecular wires in the limit of strong Coulomb repulsion. This approach is applied to two typical situations: First, we study transport through an open conduction channel for which we find that the current exhibits an ohmic-like behaviour. Second, we explore the transport properties of a bridged molecular wire, where the current decays exponentially as a function of the wire length. For both situations, we discuss the differences to the case of non-interacting electrons.
\end{abstract}

(C) 2005 Elsevier B.V. All rights reserved.

PACS: $05.60 . \mathrm{Gg} ; 85.65 .+\mathrm{h}$

Keywords: Molecular wires; Quantum transport; Coulomb repulsion

\section{Introduction}

In recent years, it became possible to adsorb organic molecules via thiol groups to a metallic gold surface and, thus, to establish a stable contact between the molecule and the gold. This opened the way to reproducible measurements of the current through single molecules. Such molecular conductance can be achieved in essentially two ways: One possible setup is an open break junction bridged by a molecule [1-3]. There, the current measurement provides evidence for single molecule conductance because asymmetries in the current-voltage characteristics reflect asymmetries of the molecule $[3,4]$. Alternatively, one can use a gold substrate as a contact and grow a self-assembled monolayer of molecules on it. The other contact is provided by a gold cluster on top of a scanning tunnelling microscope tip which contacts one or a few molecules on the substrate $[5,6]$.

\footnotetext{
* Corresponding author. Tel.: +49 821598 3250; fax: +49 8215983222. E-mail address: hanggi@physik.uni-augsburg.de (P. Hänggi).
}

Naturally, the experimental effort with such molecular wires is accompanied by a vivid theoretical interest [7-9]. Presently, the main theoretical focus lies on the ab initio computation of the orbitals relevant for the motion of excess charges through the molecular wire [10-14].

Another line of research employs rather generic models to gain a qualitative understanding of the transport mechanisms involved. The treatment of these models can be distinguished according to the level at which interaction is taken into account. Here, we are in particular interested in two situations in which the many-body problem can be traced back to the dynamics of single electrons on the wire: The first case premises non-interacting electrons for which the current can be computed from a Landauer-like formula [15-20]. The second case deals with the opposite limit in which Coulomb repulsion is so strong that at most one excess electron can be located on the molecule. Such theories have been developed in the context of conduction through coupled quantum dots [21-23] and for the incoherent transport through molecular wires [24-26].

In this work, we derive a master equation approach for molecular conduction in the limit of strong Coulomb repul- 
sion which restricts the population of the molecular orbitals to zero or one electron. Thereby, particular care will be taken in order to avoid inconsistencies like spurious non-vanishing transport in equilibrium situations. We present in Section 2 our working model and derive in Section 3 a master equation which we evaluate for the two mentioned limits, namely non-interacting electrons and strong Coulomb repulsion. Subsequently, we study in Sections 4 and 5 transport through open conduction channels and across bridged molecular wires, respectively. Explicit analytical expressions for a wire that consists of only two sites are derived in Appendix A.

\section{Model}

The setup at hand for studying coherent quantum transport is depicted in Fig. 1. The corresponding Hamiltonian reads

$H=H_{\text {wire }}+H_{\text {leads }}+H_{\text {wire-lead }}$,

where the individual terms describe the molecular wire, the electron reservoirs of the leads and the coupling of the wire to the leads. The wire itself is treated in a tight-binding approximation consisting of $N$ orbitals. Since we aim at exploring blocking effects, the corresponding wire Hamiltonian, incorporating the Coulomb repulsion in the limit of a large interaction strength $U$, assumes the form

$H_{\text {wire }}=\sum_{n} E_{n} c_{n}^{\dagger} c_{n}-\Delta \sum_{n=1}^{N-1}\left(c_{n+1}^{\dagger} c_{n}+\right.$ H.c. $)+U N(N-1)$.

The Fermion operators $c_{n}^{\dagger}\left(c_{n}\right)$ create (annihilate) an electron in the orbital $|n\rangle$ and $E_{n}$ constitutes the respective on-site energy. Here, we neglect the influence of the voltage profile on the on-site energies [27]. In the Coulomb interaction term, $N=\sum_{n} c_{n}^{\dagger} c_{n}$ is the operator counting the excess electrons on the wire. The inter-site coupling characterised by the hopping matrix element $\Delta$ is assumed to be equal between all neighbouring sites. The leads attached to the molecular wire are modelled by ideal Fermi gases,

$H_{\text {leads }}=\sum_{q} \sum_{\ell=\mathrm{L}, \mathrm{R}} \epsilon_{q} c_{\ell q}^{\dagger} c_{\ell q}$,

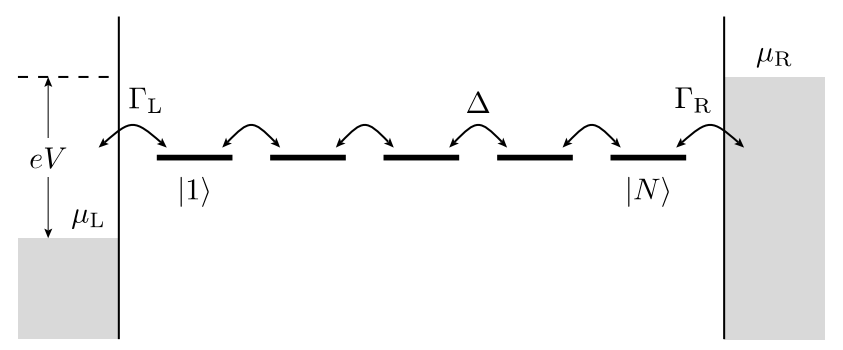

Fig. 1. Tight-binding model for an open conduction channel with $N=5$ sites. An external bias voltage $V=\left(\mu_{\mathrm{R}}-\mu_{\mathrm{L}}\right) / e$ is applied to the molecular wire. where $c_{\ell q}^{\dagger}\left(c_{\ell q}\right)$ creates (annihilates) an electron with energy $\epsilon_{q}$ in lead $\ell=\mathrm{L}, \mathrm{R}$. As an initial condition, we resort to the grand-canonical ensemble of the electrons in the leads at inverse temperature $\beta=1 / k_{\mathrm{B}} T$ and with electrochemical potentials $\mu_{\mathrm{L} / \mathrm{R}}$. Therefore, the lead electrons are described by the equilibrium Fermi function $f_{\ell}\left(\epsilon_{q}\right)=\{1+\exp$ $\left.\left[-\beta\left(\epsilon_{q}-\mu_{\ell}\right)\right]\right\}^{-1}$. For the initial density matrix, we then have

$\varrho_{\text {leads,eq }} \propto \exp \left[-\beta\left(H_{\text {leads }}-\mu_{\mathrm{L}} N_{\mathrm{L}}-\mu_{\mathrm{R}} N_{\mathrm{R}}\right)\right]$,

where $N_{\ell}=\sum_{q} c_{\ell q}^{\dagger} c_{\ell q}$ denotes the electron number in the left and right lead, respectively. From this follows that the expectation values of the lead operators can be traced back to the expression

$\left\langle c_{\ell^{\prime} q^{\prime}}^{\dagger}, c_{\ell q}\right\rangle=\delta_{\ell \ell^{\prime}} \delta_{q q^{\prime}} f_{\ell}\left(\epsilon_{q}\right)$.

The terminating sites $|1\rangle$ and $|N\rangle$, the so-called donor and acceptor sites, couple via the tunnelling matrix element $V_{\ell q}$ to the state $|\ell q\rangle$ in the respective lead. The Hamiltonian describing this interaction has the form

$H_{\text {wire-lead }}=\sum_{q}\left(V_{\mathrm{L} q} c_{\mathrm{L} q}^{\dagger} c_{1}+V_{\mathrm{R} q} c_{\mathrm{R} q}^{\dagger} c_{N}\right)+$ H.c.

It will turn out that the influence of the tunnelling matrix elements is completely characterised by the spectral density

$\Gamma_{\ell}(\epsilon)=2 \pi \sum_{q}\left|V_{\ell q}\right|^{2} \delta\left(\epsilon-\epsilon_{q}\right)$

which becomes a continuous function of $\epsilon$ if the lead modes are dense. If all relevant lead states are located in the centre of the conduction band, the energy-dependence of the spectral densities is not relevant and can be replaced by a constant, $\Gamma_{\ell}(\epsilon)=\Gamma_{\ell}$. This defines the so-called wide-band limit.

\section{Master equation approach}

The computation of stationary currents can be achieved by deriving a master equation for the dynamics of the wire electrons. Thereby, the central idea is to consider the contact Hamiltonian (6) as a perturbation. From the Liouville-von Neumann equation i $\hbar \dot{\varrho}=[H, \varrho]$ for the total density operator $\varrho$ one obtains by standard techniques [28] the approximate equation of motion

$$
\begin{aligned}
\grave{\varrho}(t)= & -\frac{\mathrm{i}}{\hbar}\left[H_{\text {wire }}(t)+H_{\text {leads }}, \varrho(t)\right] \\
& -\frac{1}{\hbar^{2}} \int_{0}^{\infty} \mathrm{d} \tau\left[H_{\text {wire-lead }},\left[\widetilde{H}_{\text {wire-lead }}(-\tau), \varrho(t)\right]\right] .
\end{aligned}
$$

The tilde denotes operators in the interaction picture with respect to the molecule and the lead Hamiltonian, $\widetilde{X}(t)=U_{0}^{\dagger}(t) X U_{0}(t)$, where $U_{0}$ is the propagator without the coupling. For the evaluation of Eq. (8) it is essential to use an exact expression for the zeroth-order time evolution operator $U_{0}(t)$. The use of any approximation bears the danger of generating artifacts, which, for instance, may lead to a violation of fundamental equilibrium properties $[28,29]$. 
The stationary current defined as the net (incoming minus outgoing) electrical current through contact $\ell$ is given by minus the time-derivative of the electron number in that lead multiplied by the electron charge $-e$ such that $I_{\ell}(t)=e(\mathrm{~d} / \mathrm{d} t)\left\langle N_{\ell}\right\rangle$. From the master equation (8) follows:

$$
\begin{aligned}
I_{\ell}(t) & =e \operatorname{tr}\left[\underline{\varrho}(t) N_{\ell}\right] \\
& =-\frac{e}{\hbar^{2}} \int_{0}^{\infty} \mathrm{d} \tau\left\langle\left[\widetilde{H}_{\text {wire-lead }}(-\tau),\left[H_{\text {wire-lead }}, N_{\ell}\right]\right]\right\rangle,
\end{aligned}
$$

where we have used the relation $\operatorname{tr} A[B, C]=\operatorname{tr}[A, B] C$. Next, we insert the wire-lead Hamiltonian (6), the interaction-picture operator $\tilde{c}_{\ell q}(-\tau)=c_{\ell q} \exp \left(\mathrm{i} \epsilon_{q} \tau\right)$ and the expectation values (5). By use of the spectral density (7), the remaining sum over the lead states is transformed into an integral which in the wide-band limit $\Gamma_{\ell}(\epsilon)=\Gamma_{\ell}$ can be evaluated to read

$$
\begin{aligned}
I_{\ell}= & \frac{e \Gamma_{\ell}}{\hbar}\left\langle c_{1}^{\dagger} c_{1}\right\rangle-e \frac{\Gamma_{\ell}}{\pi \hbar^{2}} \operatorname{Re} \int_{0}^{\infty} \mathrm{d} \tau \int \mathrm{d} \epsilon \mathrm{e}^{\mathrm{i}\left(\epsilon+\mu_{\ell}\right) \tau / \hbar} f_{l}(\epsilon) \\
& \times\left\langle\left[c_{1}, \tilde{c}_{1}^{\dagger}(-\tau)\right]_{+}\right\rangle .
\end{aligned}
$$

In the following, we specify the master equation (8) and the current formula (10) for studying two limiting cases: The first limit describes non-interacting electrons, $U=0$. For this situation, we follow the approach of [30]. The second limit is the one of strong Coulomb repulsion in which $U$ is much larger than any other energy scale of the problem. Then, only states with at most one excess electron on the wire are relevant.

In both cases, a diagonal representation of the first term on the right-hand side of the master equation (8) is achieved by a decomposition into the eigenbasis of the single-particle wire Hamiltonian. In this basis, the Fermionic interaction picture operators read

$c_{n}(t)=\sum_{\alpha}\left\langle n \mid \phi_{\alpha}\right\rangle c_{\alpha} \mathrm{e}^{-\mathrm{i} \epsilon_{\alpha} t}$,

where $\left|\phi_{\alpha}\right\rangle$ denotes an eigenstate with energy $\epsilon_{\alpha}$. Below, we will need in particular the creation and annihilation operators for the sites with direct contact to the leads, i.e. $\left|n_{\ell}\right\rangle$ where $n_{\mathrm{L}}=1$ and $n_{\mathrm{R}}=N$.

\subsection{Non-interacting electrons}

In the limit $U=0$, the transport problem defined by the Hamiltonian (1) possesses an exact solution which is conveniently derived within a scattering approach. However, since one aim of the present work is the comparison of two distinct master equations, we only sketch the exact solution for the special case of a two-level system in the Appendix A.1 and review here the corresponding master equation approach $[20,30]$.

In general, the relation between the states $\left|\phi_{\alpha}\right\rangle$ and the many-particle Hamiltonian (1) is established via the Slater determinant. Alternatively, one can resort to Green's functions. In the present case, knowledge of the Green's function at time $t=0$ is already sufficient and, besides a prefactor, equal to the expectation value

$P_{\alpha \beta}=\left\langle c_{\beta}^{\dagger} c_{\alpha}\right\rangle=P_{\beta \alpha}^{*}$.

Then, one obtains from (10) for the stationary current the expression

$I_{0}=\frac{e \Gamma_{\ell}}{\hbar} \sum_{\alpha}\left[\sum_{\beta}\left\langle\phi_{\beta} \mid n_{\ell}\right\rangle\left\langle n_{\ell} \mid \phi_{\alpha}\right\rangle P_{\alpha \beta}-\left|\left\langle n_{\ell} \mid \phi_{\alpha}\right\rangle\right|^{2} f_{\ell}\left(\epsilon_{\alpha}\right)\right]$,

where the index 0 refers to $U=0$. It can be shown that the current is independent of the index $\ell$, i.e. independent of the contact at which it is evaluated. This reflects for a two-probe setting the validity of the continuity equation.

In order to determine the expectation values $P_{\alpha \beta}$, we employ the master equation (8) and obtain for the stationary state the condition

$$
\begin{aligned}
\mathrm{i}\left(\epsilon_{\alpha}-\epsilon_{\beta}\right) P_{\alpha \beta}= & \sum_{\ell=\mathrm{L}, \mathrm{R}} \frac{\Gamma_{\ell}}{2}\left\{\left\langle\phi_{\alpha} \mid n_{\ell}\right\rangle\left\langle n_{\ell} \mid \phi_{\beta}\right\rangle\left[f_{\ell}\left(\epsilon_{\alpha}\right)+f_{\ell}\left(\epsilon_{\beta}\right)\right] .\right. \\
& -\sum_{\alpha^{\prime}}\left\langle\phi_{\alpha} \mid n_{\ell}\right\rangle\left\langle n_{\ell} \mid \phi_{\alpha^{\prime}}\right\rangle P_{\alpha^{\prime} \beta} \\
& \left.-\sum_{\beta^{\prime}}\left\langle\phi_{\beta^{\prime}} \mid n_{\ell}\right\rangle\left\langle n_{\ell} \mid \phi_{\beta}\right\rangle P_{\alpha \beta^{\prime}}\right\} .
\end{aligned}
$$

In a non-equilibrium situation, the solution of this set of equations generally possesses non-vanishing off-diagonal elements.

\subsection{Strong Coulomb repulsion}

In the limit of strong Coulomb repulsion, $U$ is assumed to be so large that at most one excess electron resides on the wire. Thus, the available Hilbert space is restricted to the states $\left\{|0\rangle, c_{\alpha}^{\dagger}|0\rangle\right\}_{\alpha=1 . . N}$, which we use for the decomposition of the density operator to obtain

$$
\begin{aligned}
\rho= & |0\rangle \rho_{00}\langle 0|+\sum_{\alpha}\left(c_{\alpha}^{\dagger}|0\rangle \rho_{\alpha, 0}\langle 0|+| 0\rangle \rho_{0 \alpha}\langle 0| c_{\alpha}\right) \\
& +\sum_{\alpha \beta} c_{\alpha}^{\dagger}|0\rangle \rho_{\alpha \beta}\langle 0| c_{\beta} .
\end{aligned}
$$

With this ansatz, the current expectation value (10) assumes the form

$$
\begin{aligned}
I_{\infty}= & \frac{e \Gamma_{\ell}}{\hbar} \sum_{\alpha}\left[\sum_{\beta}\left\langle\phi_{\beta} \mid n_{\ell}\right\rangle\left\langle n_{\ell} \mid \phi_{\alpha}\right\rangle \bar{f}_{\ell}\left(\epsilon_{\alpha}\right) \rho_{\alpha \beta}\right. \\
& \left.-\left|\left\langle\phi_{\alpha} \mid n_{\ell}\right\rangle\right|^{2} f_{\ell}\left(\epsilon_{\alpha}\right) \rho_{00}\right],
\end{aligned}
$$

where $\bar{f}=1-f$. The decomposition of the master equation (8) into the single-particle states $c_{\alpha}^{\dagger}|0\rangle$ provides for the stationary state the set of equations 


$$
\begin{aligned}
\mathrm{i}\left(\epsilon_{\alpha}-\epsilon_{\beta}\right) \rho_{\alpha \beta}= & \sum_{\ell=\mathrm{L}, \mathrm{R}} \frac{\Gamma_{\ell}}{2}\left\{\left\langle\phi_{\alpha} \mid n_{\ell}\right\rangle\left\langle n_{\ell} \mid \phi_{\beta}\right\rangle\left(f_{\ell}\left(\epsilon_{\alpha}\right)+f_{\ell}\left(\epsilon_{\beta}\right)\right) \rho_{00}\right. \\
& -\sum_{\alpha^{\prime}}\left\langle\phi_{\alpha} \mid n_{\ell}\right\rangle\left\langle n_{\ell} \mid \phi_{\alpha^{\prime}}\right\rangle \bar{f}_{\ell}\left(\epsilon_{\alpha^{\prime}}\right) \rho_{\alpha^{\prime} \beta} \\
& \left.-\sum_{\beta^{\prime}}\left\langle\phi_{\beta^{\prime}} \mid n_{\ell}\right\rangle\left\langle n_{\ell} \mid \phi_{\beta}\right\rangle \bar{f}_{\ell}\left(\epsilon_{\beta^{\prime}}\right) \rho_{\alpha \beta^{\prime}}\right\}
\end{aligned}
$$

In order to fully determine the density operator, we need in addition an expression for $\rho_{00}$ which can also be derived from the master equation. A more convenient alternative is given by the normalisation condition $\operatorname{tr} \rho=\rho_{00}+$ $\sum_{\alpha} \rho_{\alpha \alpha}=1$. For the sake of completeness, we remark that from the master equation (8) follows $\rho_{\alpha 0}=\rho_{0 \alpha}=0$ for the stationary state.

It can be shown that if the wire consists of just one site, i.e. for $N=1$, both the master equation for $U=0$ and the one for $U=\infty$ provide identical expressions for the current. The reason for this is that already the Pauli principle inhibits the occupation of the molecule by more than one electron.

\subsection{Rotating-wave approximation}

For very weak wire-lead coupling, the coherent timeevolution dominates the dynamics of the wire electrons. This means that the largest time-scale of the coherent evolution, given by the smallest energy difference, and the dissipative time-scale, determined by the coupling rates $\Gamma_{\ell}(\epsilon)$, are well-separated, i.e.,

$\Gamma_{\ell} \ll\left|\epsilon_{\alpha}-\epsilon_{\beta}\right|$

for all $\ell$ and $\alpha \neq \beta$. Note that this condition is only satisfiable if the energy spectrum has no degeneracies. Then for $\alpha \neq \beta$, Eqs. (14) and (17), which determine the stationary state, are dominated by their left-hand side. Consequently, $\rho_{\alpha \beta}$ is of the order $\Gamma /\left(\epsilon_{\alpha}-\epsilon_{\beta}\right)$ such that it can be neglected in the limit under consideration. This constitutes the essence of a rotating-wave approximation (RWA). The above reasoning is equivalent to the assumption that the stationary state is diagonal in the basis of the eigenstates. Within such a diagonal ansatz, it is possible to solve both master equations analytically and, moreover, to provide a closed expression for the respective stationary current.

\subsection{1. $R W A$ for non-interacting electrons}

In the interaction-free case, the stationary state is found by inserting the RWA ansatz $P_{\alpha \beta}=P_{\alpha \alpha} \delta_{\alpha \beta}$ into Eq. (8); after some algebra, we find

$P_{\alpha \alpha}=\frac{w_{\alpha}^{\mathrm{L}} f_{\mathrm{L}}\left(\epsilon_{\alpha}\right)+w_{\alpha}^{\mathrm{R}} f_{\mathrm{R}}\left(\epsilon_{\alpha}\right)}{w_{\alpha}^{\mathrm{L}}+w_{\alpha}^{\mathrm{R}}}$.

Thus, the populations are determined by an average over the Fermi functions, where the weights

$w_{\alpha}^{\ell}=\Gamma_{\ell}\left|\left\langle n_{\ell} \mid \phi_{\alpha}\right\rangle\right|^{2}$ are given by the overlap of the eigenstate $\left|\phi_{\alpha}\right\rangle$ with the site coupled to lead $\ell$. Then the average current is readily evaluated to read [31]

$I_{0, \mathrm{RWA}}=\frac{e}{\hbar} \sum_{\alpha} \frac{w_{\alpha}^{\mathrm{L}} w_{\alpha}^{\mathrm{R}}}{w_{\alpha}^{\mathrm{L}}+w_{\alpha}^{\mathrm{R}}}\left[f_{\mathrm{R}}\left(\epsilon_{\alpha}\right)-f_{\mathrm{L}}\left(\epsilon_{\alpha}\right)\right]$

This expression represents the limit $\Gamma \rightarrow 0$ of the corresponding scattering theory [20].

\subsubsection{RWA for strong Coulomb repulsion}

The corresponding RWA ansatz for the strongly interacting limit reads $\rho_{\alpha \beta}=\rho_{\alpha \alpha \alpha} \delta_{\alpha \beta}$. Inserting it into Eq. (17), we find the solution

$\rho_{\alpha \alpha}=\frac{1}{\mathscr{N}} \frac{w_{\alpha}^{\mathrm{L}} f_{\mathrm{L}}\left(\epsilon_{\alpha}\right)+w_{\alpha}^{\mathrm{R}} f_{\mathrm{R}}\left(\epsilon_{\alpha}\right)}{w_{\alpha}^{\mathrm{L}} \bar{f}_{\mathrm{L}}\left(\epsilon_{\alpha}\right)+w_{\alpha}^{\mathrm{R}} \bar{f}_{\mathrm{R}}\left(\epsilon_{\alpha}\right)}$,

with the weight factors $w_{\alpha}^{\ell}$ defined above and the normalisation constant

$\mathscr{N}=1+\sum_{\alpha^{\prime}} \frac{w_{\alpha^{\prime}}^{\mathrm{L}} f_{\mathrm{L}}\left(\epsilon_{\alpha^{\prime}}\right)+w_{\alpha^{\prime}}^{\mathrm{R}} f_{\mathrm{R}}\left(\epsilon_{\alpha^{\prime}}\right)}{w_{\alpha^{\prime}}^{\mathrm{L}} \bar{f}_{\mathrm{L}}\left(\epsilon_{\alpha^{\prime}}\right)+w_{\alpha^{\prime}}^{\mathrm{R}} \bar{f}_{\mathrm{R}}\left(\epsilon_{\alpha^{\prime}}\right)}$.

The average current follows directly by inserting $\rho_{\alpha \alpha}$ into (16) and reads

$I_{\infty, \mathrm{RWA}}=\frac{e}{\hbar \mathscr{N}} \sum_{\alpha} \frac{w_{\alpha}^{\mathrm{L}} w_{\alpha}^{\mathrm{R}}}{w_{\alpha}^{\mathrm{L}} \bar{f}_{\mathrm{L}}\left(\epsilon_{\alpha}\right)+w_{\alpha}^{\mathrm{R}} \bar{f}_{\mathrm{R}}\left(\epsilon_{\alpha}\right)}\left[f_{\mathrm{R}}\left(\epsilon_{\alpha}\right)-f_{\mathrm{L}}\left(\epsilon_{\alpha}\right)\right]$.

This current formula differs from the one obtained within RWA for non-interacting electrons, Eq. (21), by the appearance of the normalisation factor $\mathscr{N}$ and by the Fermi functions $\bar{f}=1-f$ in the denominator.

\section{Open transport channel}

As a first application, we consider a wire for which all on-site energies are at the level of the chemical potentials and, moreover, all hopping matrix elements are equal, as sketched in Fig. 1. Then, the molecular orbitals are delocalised which provides ideal transport along the molecule. For a voltage which is sufficiently large such that all molecular orbitals lie within the voltage window, the current is in the interaction-free case dominated by the total transmission. Under the assumption that all overlaps (20) between molecule eigenstates and sites $|n\rangle$ are identical, we find $w_{\alpha}^{\ell}=\Gamma / N$. Then, the RWA current formula (21) becomes

$I_{0}=\frac{e \Gamma}{2 \hbar}$.

In particular, we find that the current is independent of the wire length which is characteristic for coherent transport of non-interacting electrons [32].

This behaviour is significantly modified by the influence of strong Coulomb repulsion: Then one finds that the normalisation factor (23) reads $\mathscr{N}=N+1$ such that finally

$I_{\infty}=\frac{e \Gamma}{\hbar(N+1)}$. 


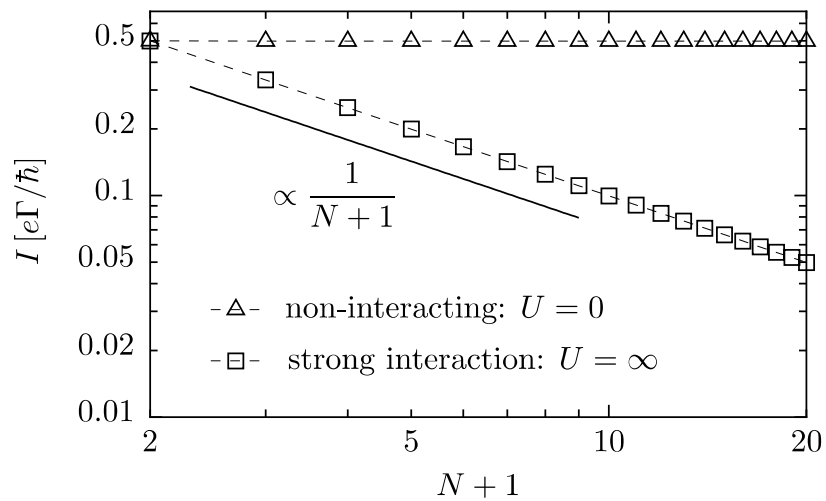

Fig. 2. Stationary current as a function of the wire length for the transport through the open channel sketched in Fig. 1 with bias voltage $V=10 \Delta / \hbar$. The other parameters are $\Gamma=0.1 \Delta$ and $k_{\mathrm{B}} T=0.005 \Delta$. The dashed lines are a guide to the eye.

For $N=1$, this result coincides with Eq. (25), as expected. For a long wire, we find that the current decreases $\propto 1 / N$ i.e. with the inverse of the wire length. This behaviour resembles an ohmic resistor and has been observed in the limit of strong Coulomb repulsion also for incoherent hopping of the wire electron [26].

The numerically computed current beyond RWA is shown in Fig. 2. It fully confirms the respective length dependence and, moreover, demonstrates the applicability of the rotating-wave approximation in the present case.

\section{Bridged molecular wire}

Next, we consider the bridged molecular wire model sketched in Fig. 3. There, the energies of the donor and the acceptor orbitals, $|1\rangle$ and $|N\rangle$, are assumed to be close to the chemical potentials of the attached leads, $\mu_{\mathrm{L}} \lesssim E_{1}=E_{N} \lesssim \mu_{\mathrm{R}}$. The bridge levels $E_{n}, n=2, \ldots$, $N-1$, lie $E_{\mathrm{B}} \gg \Delta$ above the chemical potential.

Let us first discuss the eigenstates of the molecule which discern into two groups: One group of states is located on the bridge. It consists of $N-2$ levels with energies in the range $\left[E_{\mathrm{B}}-2 \Delta, E_{\mathrm{B}}+2 \Delta\right]$. In the absence of the driving field, these bridge states mediate the super-exchange between the donor and the acceptor. The other group consists of the two remaining states. They form a doublet whose

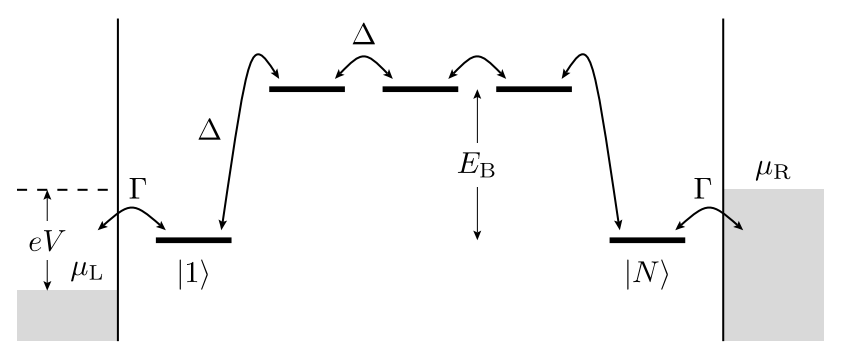

Fig. 3. Level structure of the bridged molecular wire with $N=5$ sites. The bridge levels are separated by $E_{\mathrm{B}}$ from the donor and acceptor levels $|1\rangle$ and $|N\rangle$. states are approximately given by $(|1\rangle \pm|N\rangle) / \sqrt{2}$. Its splitting can be estimated in a perturbational approach [33] and is approximately given by $2 \Delta\left(\Delta / E_{\mathrm{B}}\right)^{N-2}$. Thus, the wire can be reduced to a two-level system with the effective tunnel matrix element $\Delta_{\mathrm{DA}}=\Delta \exp [-\kappa(N-2)]$, where $\kappa=\ln \left(E_{\mathrm{B}} / \Delta\right)$. The explicit calculation for the two-level system is given in Appendix A.

For non-interacting electrons, we find from (A.4) to lowest order in $\Delta_{\mathrm{DA}} / \Gamma$ by replacing $\Delta \rightarrow \Delta_{\mathrm{DA}}$

$I_{0}=\frac{2 e|\Delta|^{2}}{\hbar \Gamma} \mathrm{e}^{-2 \kappa(N-2)}$,

while in the case of strong Coulomb repulsion we employ (A.13) to obtain

$I_{\infty}=\frac{4 e|\Delta|^{2}}{\hbar \Gamma} \mathrm{e}^{-2 \kappa(N-2)}$.

In particular, one finds in both cases an exponentially decaying length dependence of the current $[7,15]$. Quite remarkably, the current in the strongly interacting case is larger by a factor 2 .

In order to test the quality of the two-level approximation above, we compare the analytical result (28) against the numerical solution of the respective master equation. Fig. 4 demonstrates the almost perfect agreement between the numerical and the analytical solution. Moreover, it confirms the exponentially decaying length dependence and the enhancement of the current by a factor 2 owing to Coulomb interaction. For $N=3$, the limit $\Delta_{\mathrm{DA}} \ll \Gamma$ is not yet reached which explains the small deviation from expression (28).

We close this section with the remark that for bridged molecular wires, the rotating-wave approximation derived in Section 3.3 results in $I_{0}=e \Gamma / 2 \hbar$ and $I_{\infty}=e \Gamma / 3 \hbar$. Thus, the RWA even fails to predict the observed length dependence qualitatively. The reason for this is that $\Delta_{\mathrm{DA}} \ll \Gamma$ and, thus, the condition (18) for the applicability of the RWA is violated.

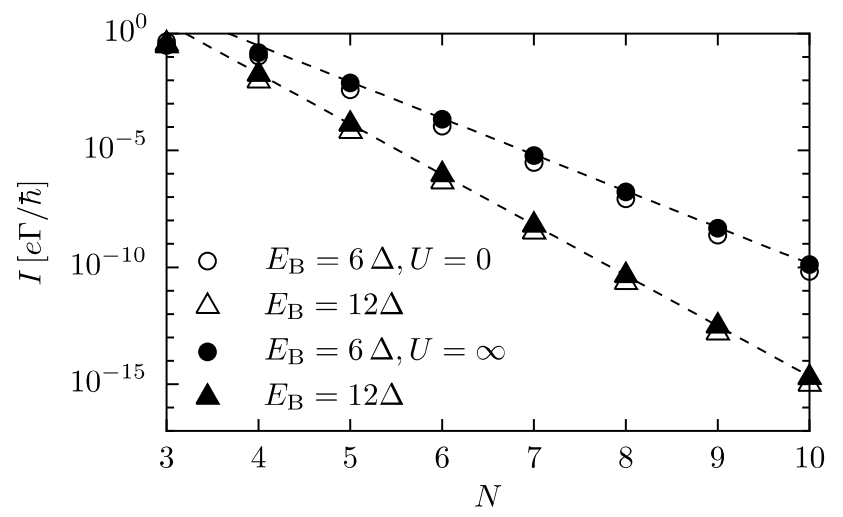

Fig. 4. Current for the bridged molecular wire model, cf. Fig. 3 comparing the non-interacting case with strong Coulomb repulsion. The bias voltage is $V=5 \Delta / \hbar, \Gamma=0.1 \Delta$, and $k_{\mathrm{B}} T=0.005 \Delta$. The dashed lines mark the analytical result (28) for $U=\infty$. 


\section{Conclusions}

We have derived a master equation approach for the electron transport through tight-binding systems in the presence of strong Coulomb repulsion. In contrast to prior work, we treat the master equation beyond a rotating-wave approximation which enlarges the range of validity of our approach. In particular, we have presented an example for which our approach provides reliable results while within a rotating-wave approximation one obtains qualitatively wrong results.

With this formalism, we have studied transport properties of two models for molecular wires. Thereby, we have worked out the differences to the case of non-interacting electrons. A model for which all on-site energies are identical, represents a tight-binding version of an open conduction channel. There, we find a significant influence of Coulomb repulsion: While in the absence of interaction, the current is length independent, it decreases due to Coulomb repulsion proportional to the wire length. Thereby it resembles an ohmic conductor even though the transport is fully coherent.

For the bridged molecular wire model, only the first and the last site have energies close to the chemical potentials of the leads. Then, all the other levels merely mediate co-tunnelling. We have demonstrated that then the wire exhibits the behaviour of a two-level system. In particular, we found the surprising effect that Coulomb blocking enhances the current by a factor two.

Comparing the results for the open-channel model and the bridge model, we can conclude that the influence of Coulomb repulsion depends sensitively on the level structure of the molecule. In particular if many unoccupied molecular orbitals have energies close to the chemical potentials of the leads, electron-electron interaction reduces the current considerably.

\section{Acknowledgements}

The authors acknowledge financial support by the Deutsche Forschungsgemeinschaft through Graduiertenkolleg 283 and Sonderforschungsbereich 486. One of us (P.H.) acknowledges many elucidative, advisable and inspiring scientific discussions with Philip Pechukas, who is still young and energetic enough to contribute to great science and literature.

\section{Appendix A. Two-level system}

The bridged molecular wire discussed in Section 5 can be described by a wire that consists of only a donor state $|1\rangle$ and an acceptor state $|2\rangle$, i.e. $N=2$. These two sites are coupled by an effective tunnel matrix element $\Delta$. In this appendix, we derive explicit results for the transport through an unbiased two-level system $\left(E_{1}=E_{2}=0\right)$ for $\Gamma_{\mathrm{L}}=\Gamma_{\mathrm{R}}=\Gamma$ and chemical potentials such that effectively $f_{\mathrm{L}}=0$ and $f_{\mathrm{R}}=1$. Diagonalising the wire Hamiltonian
(2) for vanishing Coulomb interaction $(U=0)$, we obtain the bonding and anti-bonding eigenstates and eigenenergies

$$
\begin{aligned}
& \left|\phi_{+}\right\rangle=\frac{1}{\sqrt{2}}(|1\rangle+|2\rangle), \quad \epsilon_{+}=-\Delta, \\
& \left|\phi_{-}\right\rangle=\frac{1}{\sqrt{2}}(|1\rangle-|2\rangle), \quad \epsilon_{-}=\Delta .
\end{aligned}
$$

For weak coupling between the donor and the acceptor, $\Delta \ll \Gamma$, the master equation approach, albeit perturbational in $\Gamma$, still provides the correct behaviour owing to the proper inclusion of off-diagonal elements of the density matrix. Within rotating-wave approximation this is no longer the case.

\section{A.1. Landauer form}

According to Landauer [34], the coherent transport for non-interacting electrons can be interpreted as a quantum mechanical scattering process. Thereby the in- and outgoing electronic states scattered in the mesoscopic conductor are considered as plane waves. As a consequence, the pivotal quantity which determines the system's conductance is the transmission probability $T(E)$ and the corresponding current can be written in the form

$I_{0}=\frac{e}{2 \pi \hbar} \int \mathrm{d} E\left[f_{\mathrm{R}}(E)-f_{\mathrm{L}}(E)\right] T(E)$.

The transmission can now be calculated via the relation $T(E)=\Gamma_{\mathrm{L}} \Gamma_{\mathrm{R}}\left|G_{12}(E)\right|^{2}$, where $G(E)=\left(E-H_{\text {wire }}-\mathrm{i} \Gamma / 2\right)^{-1}$ denotes the retarded Green's function.

For an unbiased two-level system, we obtain

$T(E)=\frac{\Gamma^{2} \Delta^{2}}{\left|(E-\mathrm{i} \Gamma / 2)-\Delta^{2}\right|^{2}}$.

Inserting this expression into Eq. (A.2), one arrives at

$I_{0}=\frac{e \Gamma}{2 \hbar} \frac{\Delta^{2}}{\Delta^{2}+(\Gamma / 2)^{2}}$.

A more explicit calculation can be found, e.g., in [35].

\section{A.2. Non-interacting electrons}

For the eigenstates and eigenenergies (A.1), the current formula (13), valid for $U=0$, reads $I_{0}=(e \Gamma / 2 \hbar) \Sigma_{\alpha, \beta} P_{\alpha \beta}$ while the set of Eqs. (14) becomes:

$$
\begin{aligned}
& 0=\frac{\Gamma}{2}\left(1-2 P_{++}\right), \\
& 0=\frac{\Gamma}{2}\left(1-2 P_{--}\right), \\
& -2 \mathrm{i} \Delta P_{+-}=\frac{\Gamma}{2}\left(1-2 P_{+-}\right) .
\end{aligned}
$$

This corresponds to $P_{++}=P_{--}=1 / 2$ and $P_{+-}=\Gamma /$ $(2 \Gamma-4 \mathrm{i} \Delta)=P^{*}{ }_{-+}$. Inserting this solution into Eq. (13), we obtain the stationary current (A.4). 
The quality of the present master equation approach is underlined by the fact that it here indeed reproduces even for $\Gamma \gg \Delta$ the exact solution. We emphasise that this is not the case for the RWA solution (21): Since for this approximation by definition $P_{+_{-}}=0$, one obtains the result $I_{0, \mathrm{RWA}}=e \Gamma / 2 \hbar$ which is independent of the inter-site coupling $\Delta$.

\section{A.3. Strong Coulomb repulsion}

Using the eigenstates and eigenenergies (A.1), one finds that the current with Coulomb blocking, Eq. (16), reads $I_{\infty}=(e \Gamma / 2 \hbar) \sum_{\alpha, \beta} \rho_{\alpha \beta}$. Formally, this is identical to the corresponding expression for the non-interacting case but with the $P_{\alpha \beta}$ replaced by the matrix elements of the density operator in the basis of the single particle states $c_{\alpha}^{+}|0\rangle$. The stationary solution of these matrix elements is determined by Eq. (17) which for a two-level system becomes

$0=\frac{\Gamma}{2}\left(1-2 \rho_{++}-\rho_{--}-\operatorname{Re} \rho_{+-}\right)$,

$0=\frac{\Gamma}{2}\left(1-2 \rho_{--}-\rho_{++}-\operatorname{Re} \rho_{+-}\right)$,

$-2 \mathrm{i} \Delta \rho_{+-}=\frac{\Gamma}{2}\left(1-\frac{1}{2} \rho_{++}-\frac{1}{2} \rho_{--}+\rho_{+-}\right)$.

This corresponds to the solution

$\rho_{++}=\rho_{--}=\frac{8 \Delta^{2}+\Gamma^{2}}{2\left(12 \Delta^{2}+\Gamma^{2}\right)}$,

$\rho_{+-}=\rho_{-+}^{*}=-\frac{4 \Delta \mathrm{i} \Gamma+\Gamma^{2}}{2\left(12 \Delta^{2}+\Gamma^{2}\right)}$.

Inserting into Eq. (16), we obtain the current

$I_{\infty}=\frac{e \Gamma}{2 \hbar} \frac{2 \Delta^{2}}{3 \Delta^{2}+(\Gamma / 2)^{2}}$.

\section{References}

[1] M.A. Reed, C. Zhou, C.J. Muller, T.P. Burgin, J.M. Tour, Science 278 (1997) 252.

[2] C. Kergueris, J.-P. Bourgoin, S. Palacin, D. Esteve, C. Urbina, M. Magoga, C. Joachim, Phys. Rev. B 59 (1999) 12505.
[3] J. Reichert, R. Ochs, D. Beckmann, H.B. Weber, M. Mayor, H. von Löhneysen, Phys. Rev. Lett. 88 (2002) 176804.

[4] H.B. Weber, J. Reichert, F. Weigend, R. Ochs, D. Beckmann, M. Mayor, R. Ahlrichs, H. von Löhneysen, Chem. Phys. 281 (2002) 113.

[5] S. Datta, W. Tian, S. Hong, R. Reifenberger, J.I. Henderson, C.P. Kubiak, Phys. Rev. Lett. 79 (1997) 2530.

[6] X.D. Cui, A. Primak, X. Zarate, J. Tomfohr, O.F. Sankey, A.L. Moore, T.A. Moore, D. Gust, G. Harris, S.M. Lindsay, Science 294 (2001) 571.

[7] A. Nitzan, Annu. Rev. Phys. Chem. 52 (2001) 681.

[8] P. Hänggi, M. Ratner, S. Yaliraki, Chem. Phys. 281 (2002) 111.

[9] A. Nitzan, M.A. Ratner, Science 300 (2003) 1384.

[10] M. Di Ventra, S.T. Pantelides, N.D. Lang, Phys. Rev. Lett. 84 (2000) 979.

[11] Y. Xue, S. Datta, M.A. Ratner, Chem. Phys. 281 (2002) 151.

[12] P. Damle, A.W. Ghosh, S. Datta, Chem. Phys. 281 (2002) 171.

[13] J. Heurich, J.C. Cuevas, W. Wenzel, G. Schön, Phys. Rev. Lett. 88 (2002) 256803.

[14] F. Evers, F. Weigend, M. Koentopp, Phys. Rev. B 69 (2004) 235411.

[15] V. Mujica, M. Kemp, M.A. Ratner, J. Chem. Phys. 101 (1994) 6849.

[16] D. Segal, A. Nitzan, W.B. Davis, M.R. Wasielewski, M.A. Ratner, J. Phys. Chem. 104 (2000) 3817.

[17] L.E.F. Foa Torres, H.M. Pastawski, S.S. Makler, Phys. Rev. B 64 (2001) 193304.

[18] M. Čížek, M. Thoss, W. Domcke, Phys. Rev. B 70 (2004) 125406.

[19] M. del Valle, C. Tejedor, G. Cuniberti, Phys. Rev. B 71 (2005) 125306.

[20] S. Kohler, J. Lehmann, P. Hänggi, Phys. Rep. 406 (2005) 379.

[21] T.H. Stoof, Yu. V. Nazarov, Phys. Rev. B 53 (1996) 1050.

[22] T. Brandes, R. Aguado, G. Platero, Phys. Rev. B 69 (2004) 205326.

[23] C. Flindt, T. Novotný, A.-P. Jauho, Phys. Rev. B 70 (2004) 205334.

[24] E.G. Petrov, P. Hänggi, Phys. Rev. Lett. 86 (2001) 2862.

[25] E.G. Petrov, V. May, P. Hänggi, Chem. Phys. 281 (2002) 211.

[26] J. Lehmann, G.-L. Ingold, P. Hänggi, Chem. Phys. 281 (2002) 199.

[27] A. Nitzan, M. Galperin, G.-L. Ingold, H. Grabert, J. Chem. Phys. 117 (2002) 10837.

[28] V. May, O. Kühn, Charge and Energy Transfer Dynamics in Molecular Systems, second ed., Wiley-VCH, Weinheim, 2003.

[29] T. Novotný, Europhys. Lett. 59 (2002) 648.

[30] J. Lehmann, S. Kohler, P. Hänggi, A. Nitzan, Phys. Rev. Lett. 88 (2002) 228305.

[31] J. Lehmann, S. Kohler, P. Hänggi, A. Nitzan, J. Chem. Phys. 118 (2003) 3283.

[32] S. Datta, Electronic Transport in Mesoscopic Systems, Cambridge University Press, Cambridge, 1995.

[33] M.A. Ratner, J. Phys. Chem. 94 (1990) 4877.

[34] R. Landauer, IBM J. Res. Dev. 1 (1957) 223.

[35] S. Kohler, S. Camalet, M. Strass, J. Lehmann, G.-L. Ingold, P. Hänggi, Chem. Phys. 296 (2004) 243. 